
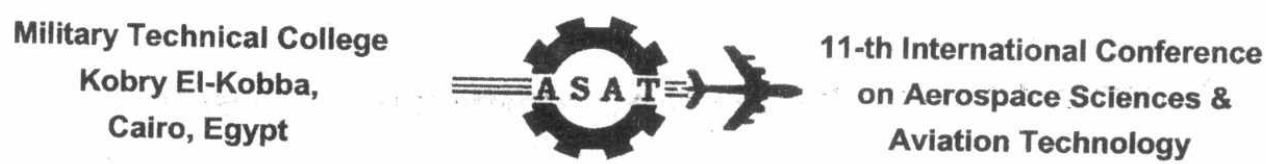

\title{
NEW RATIO IMPROVING THE DIAGNOSIS OF NERVE SYNDROME
}

\author{
Wael Farouk, Elsayed Abd-Alaziz Sleet, Serry.S.Besar, M.EL.Sayed Gadallah, \\ Ahmed Genedy
}

\begin{abstract}
Sensory nerve action potential is always characterized by three factor, amplitude, duration and waveform. In this paper we proposed an additive ratio (median to ulnar sensory nerve action potential amplitudes ratio) for improving the diagnosis of nerve syndrome while measuring sensory nerve action potential (SNAP). Orthodromic median and ulnar SNAPs were recorded and the respective ratios were calculated. Normative data for median nerve SNAP amplitude, ulnar nerve SNAP amplitude and the new ratio is obtained.
\end{abstract}

\section{KEYWORDS}

Sensory nerve action potential; median nerve; ulnar nerve; ENG recording.

\section{1-INTRODUCTION}

The sensory nerve action potential (SNAP) always characterized by its amplitude, duration, and waveform [1]. The amplitude of the SNAP is measured from the peak of the positive deflection to the peak of the negative deflection in microvolt. Moreover, this amplitude depends on the number of axons being stimulated and the synchrony with which they transmit their impulse. If the axons transmit impulses at comparable velocities, the response duration will be short with amplitude high. However, if the axonal velocities are widely dispersed, the SNAP duration will be longer and its amplitude will be lower. It was found that the variability of median and ulnar sensory nerve action potentials is great [2]. The amplitude of SNAP is also related to temperature, digit size, electrode size, electrode material, and electrode placement. To comment on the clinical value of a diagnostic test, it is imperative to have a gold standard. Some investigators have used clinical history and physical examination as the gold standard. Our aim in this study to get a gold standard for our laboratory during measuring sensory nerve action potential, by adding the ratio (the median to ulnar sensory nerve action potential amplitude ratio) to improve the diagnosis of nerve neuropathy. 


\section{2-SYSTEM CONSTRUCTION}

The system used in recording electroneurogram signal (ENG) consists of the electrode, amplifier, ADC, stimulator and display as shown in fig.1

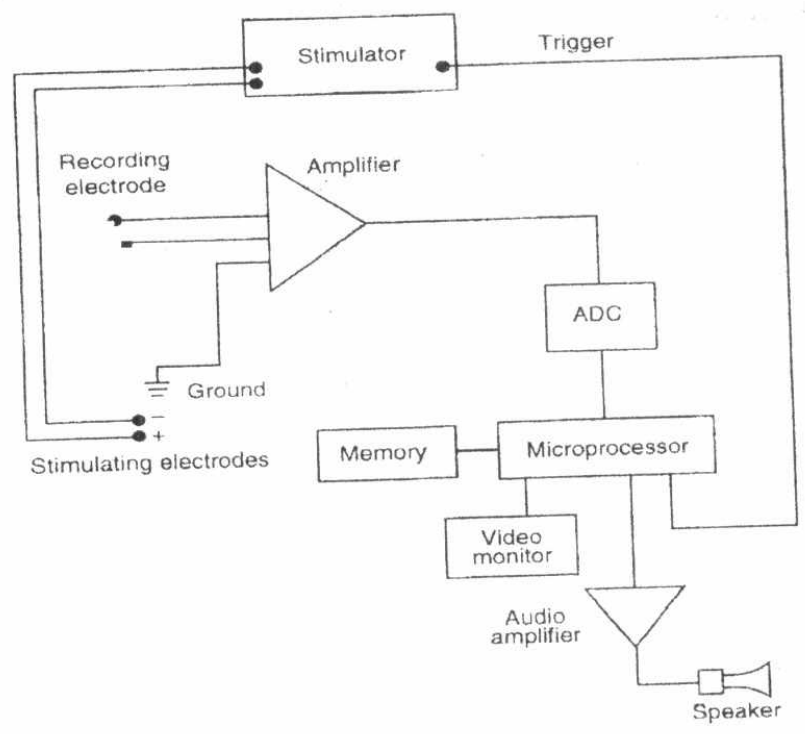

Fig.1. Block diagram of the system used in recording electroneurogram (ENG) signal.

\section{2-1 Electrodes}

Electrodes are used in studies of nerve conduction velocity and neuromuscular transmission, and whenever it is necessary for other reasons to determine the characteristics of the electrical response of a muscle to stimulation of its motor nerve [3]. The potential that is recorded represents the sum of the individual potentials produced by all of the nerve or muscle fibers that are activated [4]. When surface electrodes are used, the impedance between them and the skin must be reduced. These electrodes usually consist of metal disc, varying in diameter from 0.5 to $2.5 \mathrm{~cm}$, which are attached individually to the patient [5]. In practice, the potential difference between two suitably placed electrodes is recorded by connecting each to one side of the differential amplifier. A third electrode is used to connect the patient with ground. It is important to note the sites at which active and reference electrodes are placed will influence the amplitude and waveform of the potentials that are recorded. In this study we used a ring electrode shown in fig. 2 as a stimulation electrode surrounded the fourth digit to stimulate both median and ulnar nerve at the wrist. Surface electrode was used with the configuration studied in [6] to record the sensory nerve action potential for both median and ulnar nerve. 


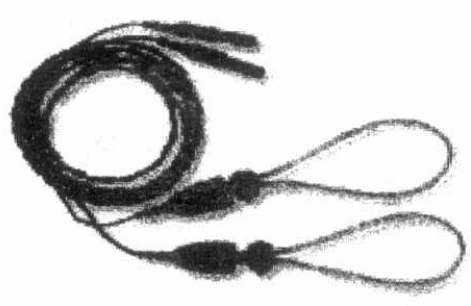

Fig.2. Photo of the ring electrode used to stimulate median and ulnar nerve

\subsection{Amplifier and Preamplifier}

Since the used electrodes pick up various interference signals, as well as the desired bioelectric activity. The bioelectric activity should be enhanced. This is accomplished by a preamplifier that is placed relatively close to the electrodes.

The bioelectric potentials must be preamplifier before they can be displayed or recorded satisfactorily. By using a differential amplifier, it is possible to achieve this while simultaneously rejecting any interference signals [7]. it amplifies differences that exists in the signals from its two inputs, but rejects signals that are common to both inputs, such as those caused by interference. The ability of an amplifier to reject such common mode signals is indicated by its common mode rejection ratio (CMRR) [8].

CMRR $(\mathrm{dB})=20 \log$ (gain of differences signal / gain of common mode signal)

The higher this ratio is, the greater the ability of the amplifier to reject common mode potentials.

Another important characteristic of the amplifier is the ratio of its output voltage to its input voltage.

Gain $(\mathrm{dB})=20 \log$ (output voltage/input voltage)

Depending on the type of investigation being conducted, it is sometimes necessary to alter the frequency response of the amplifier by the use of filters [9]. These filters eliminate or attenuate noise and interference signals with a different frequency from that of the bioelectric signals being observed [10].

\subsection{Stimulator}

For recording the ENG signal, it's necessary to stimulate the nerve electrically. For this purpose, a generator is used to pass a rectangular pulse of current for a brief period. The duration of this stimulus is usually adjustable, ranging from 0.05 to 1 msec. The current is generally passed through two surface electrodes that are placed in such a way that the current flow between them passes through the nerve to be stimulated. The resistance of the dry skin to the flow of an electric current is reduced by lightly covering the electrode with electrode jelly. 


\section{3 -MATERIAL AND METHOD}

Thirty five healthy male and twenty two healthy female volunteers without neurological complaints were studied to get gold standard for normality reading of the SNAP and the new ratio (median to ulnar sensory nerve action potential amplitude ratio). Orthodromic median and ulnar SNAPs were recorded and the new ratio was calculated. All subjects were tested in the same room by the same technique.

The electrodes were applied to the right wrist of the subject and positioned first over the median nerve at the wrist. The active electrode was placed distally with the most distal margin of the wrist. No change of the electrode location took place after its initial placement unless the baseline was not stable or if the stimulus artifact disturbed it.

Supramaximal stimulation was applied by ring electrodes, covering with electrode gel encircling the forth finger of the right hand to stimulate both median and ulnar nerve see fig. 3 .

The stimulation current with an amplitude of $10 \mathrm{~mA}$, and frequency $1 \mathrm{sec}$ and a duration of 100usec was used and the results were averaged. The amplitude was then measured. A total of 3 tests of 100 stimuli each were made before the electrode removed. And this is a one trial. The amplitude for each trial was therefore the mean of the 3 averaged SNAP amplitude of 100 stimuli each. The recording electrode was then re-position to the place over the ulnar nerve and repeat the measurements. For each trial the amplitude of the SNAP for the median and ulnar nerve were determined. The ratio of the median nerve SNAP amplitude to ulnar nerve SNAP amplitude was determined.

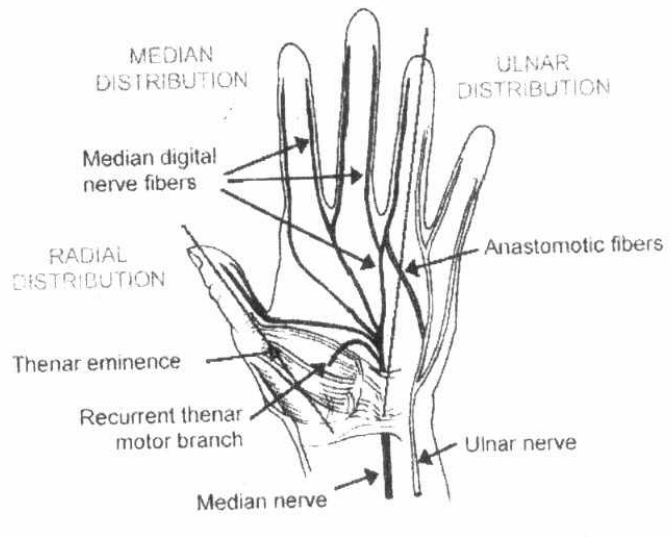

Fig.3. Distribution of Median and Ulnar Nerve.

\section{4- RESULTS}

After completing our measurements, it was found that the median SNAP amplitude values ranged from a minimum value of $6.5 \mu \mathrm{V}$ to a maximum value of $26.3 \mu \mathrm{V}$, The 
ulnar SNAP amplitude values ranged from a minimum value of $5 \mu \mathrm{V}$ to a maximum value of $21.4 \mu \mathrm{V}$. The new ratio values ranged from a minimum value of $0.69 \mu \mathrm{V}$ to a maximum value of $2.9 \mu \mathrm{V}$, see Table 1 .

Table 1 the minimum and maximum values of the median nerve SNAP amplitude, ulnar nerve SNAP amplitude, new ratio estimated from a group of normal people (thirty five male, twenty two female).

\begin{tabular}{|c|c|c|c|}
\hline Sensory values & $\begin{array}{c}\text { Median nerve } \\
\text { amplitude }(\mu V)\end{array}$ & $\begin{array}{c}\text { Ulnar nerve } \\
\text { amplitude }(\mu V)\end{array}$ & The new ratio \\
\hline Max. & 26.3 & 21.4 & 2.9 \\
\hline Min. & 6.5 & 5 & 0.69 \\
\hline
\end{tabular}

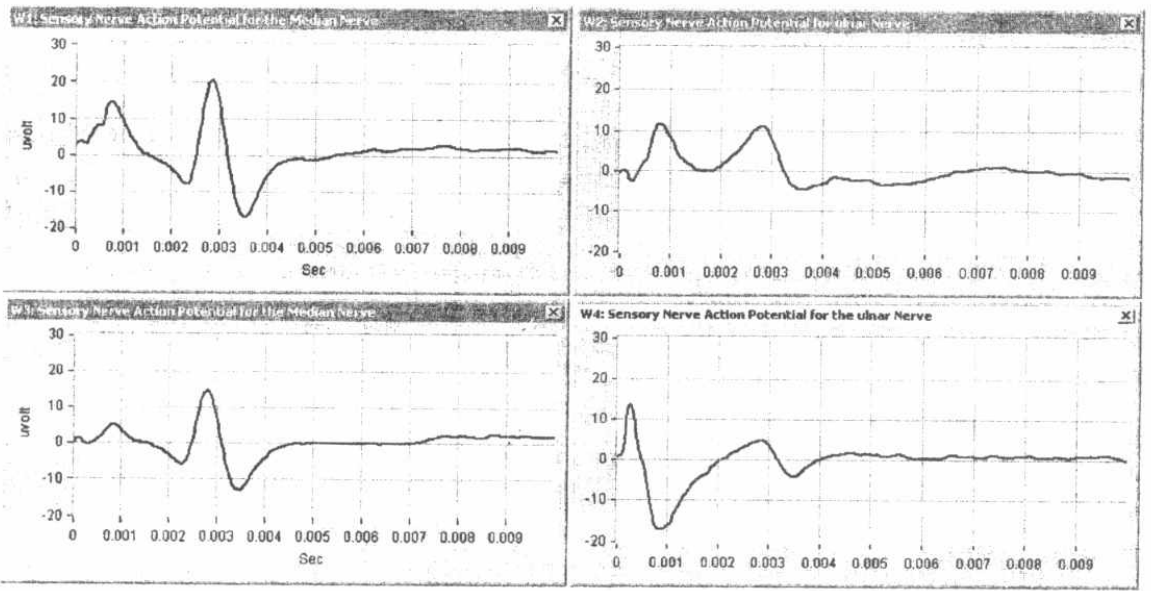

Fig.4 The figure in the upper and lower left hand side represents a sample of the SNAP for the median nerve of a man and women respectively, while the upper and lower right figures represents the SNAP for the ulnar nerve.

\section{5- CONCLUSION}

In this paper we proposed new ratio to be used in clinical work which will be promising in decreasing the error in diagnosing nerve syndrome. Furthermore, it will increase the diagnostic specificity. We achieved a gold standard for our laboratory from a number of normal patients and specified the normal ranges for the median and ulnar nerve sensory action potential amplitudes. Moreover we estimated the proposed new ratio and specified its normal range to facilitate the differentiation between normal and abnormal. 


\section{REFERENCES}

[1] Meythaler JM et.al. "Electrophysiologic analysis of SNAP amplitude in orthodromic and antidromic studies". Electromyography Clinical Neurophysiology. 1994.

[2] Kimura J. "Electrodiagnosis in the diseases of nerves and muscles". Philadelphia (PA): FA Davis; 1984.

[3] G.Morita et.al "Estimation of the conduction velocity distribution of human sensory nerve fibers" journal of electromyography and Kinesiology 12(2002) $37-43$.

[4] David H.Liang, et.al. "The Nerve-Electrode Interface of The Cochlear Implant: Current Spread" IEEE Transactions on biomedical engineering January 1999.

[5] EMG Electrodelwww.delsys.comiproductslelectrodes.htm.

[6] W.Farouk et.al "Non Invasive Technique for Studying the effect of electrode Geometry on Interference Reduction during measuring sensory nerve action potential" fourth international conference on electrical engineering November 2004.

[7] Zoran M.Nikolie et.al."Instrumentation for ENG and EMG recording in FES systems". IEEE Transactions on biomedical engineering vol.41 july1994.

[8] Ramon Pallas-Areny "Interference rejection characteristics of biopotential amplifier: A comparative analysis" IEEE Transactions on biomedical engineering November 1988.

[9] Martin D.Wells and Shai N. Gozani "A Method to Improve The Estimation of Conduction Velocity Distribution Over A Short Segment of Nerve" IEEE Transactions on biomedical engineering September 1999.

[10] E.A. Clancy , E.L. Morin b, R. Merletti "Sampling, noise-reduction and amplitude estimation issues in Surface electromyography Journal of Electromyography and Kinesiology 12 (2002). 







\section{CONTENTS}

Index of Authors

Index of Keywords

Index of Reviewers 